\title{
Bayesian Network Approach to Customer Requirements to Customized Product Model
}

\author{
Qin Yang $\mathbb{D}^{1},{ }^{1}$ Zhirui Li $\left(\mathbb{D},{ }^{1}\right.$ Haisen Jiao, ${ }^{1}$ Zufang Zhang, ${ }^{1,2}$ Weijie Chang, ${ }^{1}$ and Daozhu Wei ${ }^{1}$ \\ ${ }^{1}$ School of Mechanical Engineering, Hefei University of Technology, Hefei230009, China \\ ${ }^{2}$ Hefei University of Tecnology (Maanshan) High-technology Institute, Maanshan243000, China
}

Correspondence should be addressed to Qin Yang; yangqin@hfut.edu.cn

Received 15 April 2019; Accepted 2 June 2019; Published 15 July 2019

Academic Editor: Paolo Renna

Copyright (C) 2019 Qin Yang et al. This is an open access article distributed under the Creative Commons Attribution License, which permits unrestricted use, distribution, and reproduction in any medium, provided the original work is properly cited.

Customizing products based on customer needs is an irreversible trend, and many companies strive to provide customized products to customers in less time. Customer requirements are a key factor in the company's ability to provide customized products. In order to better meet customer needs, solve the problem of incomplete and inaccurate expression, and improve the correlation between customized product performance and customer demand, a customized product method based on Bayesian network is proposed. First, the company built a custom product model based on Bayesian networks. According to the model, the customer selects some nodes and their related information. Then, we can accurately predict the final product model through the link tree algorithm, test the customer demand node to determine the focus of the customer's needs, and optimize the model. Finally, an example of a multifunctional nursing bed is used to illustrate the effectiveness of the method.

\section{Introduction}

In order to provide customized products, the company's urgent need is to obtain simple and accurate customer information, which can be used to predict product configuration to obtain satisfactory product configuration solutions $[1,2]$. The purpose of product configuration design is to meet the individual needs of customers [3]. The customer requirements are transformed into the form of demand nodes and demand expressions as the basis of the customized product model. The ant colony optimization algorithm is used to optimize the search expression path and obtain complete customer requirements [4] so the acquisition and analysis of customer needs have a great impact on the final product. Customer requirements refer to customer preferences or descriptions of the product, including functionality, appearance, and cost. Clear and complete customer requirements help companies develop products with higher customer satisfaction, reduce time-to-market, help manufacturers capture larger markets, and achieve sustainable growth. As a result, customer demand has become a key factor in increasing competition, and most companies are aware of the importance of customer demand. However, because most customers lack product expertise and demand expression skills are weak, customer requirements are always unclear and incomplete. The reasons are summarized as follows:

(1) Due to the lack of knowledge in the professional field, the customer's expression is not accurate enough. For example, customers do not understand car displacement and turbochargers, which will lead to wrong customer requirements.

(2) Due to the inaccuracy of customer decision-making and the limitation of expressive ability, people always use natural language to describe the weight of product parameters or technical features, such as "price should not be too high."

(3) Customer requirements are dynamic $[5,6]$, and people's preferences and requirements can change over time.

For the above reasons, it is difficult for companies to obtain and understand real customer needs. The difficulty of obtaining customer demand reduces the accuracy of product configuration forecasts. How to solve this problem is the focus of current research. There are two main ways to solve it. The first method is to start with the expression of customer needs and make the expression more complete and accurate. For example, Nahm et al. [7] proposed an evaluation method for the importance of customer requirements in 
quality function deployment (QFD), which improved the accuracy of demand conversion. Chen et al. [8] proposed an ontology learning customer demand representation system. In this system, a customer demand ontology is established to generate a more accurate demand report. Sheng et al. [9] proposed the customer demand modeling and mapping process for configuration design. Ambiguity and abstraction requirements are decomposed and then converted by QFD. The decomposition rules are given. Carulli et al. [10] proposed a method based on virtual reality technology to capture the characteristics of products. It allows companies to collect product configuration preferences at the beginning of the product development process. Aguwa et al. [11] convert different forms of customer input (including qualitative and quantitative data) into a common data format for interpreting and analyzing customer sounds. But these solutions often still require some accurate customer demand information, and they cannot solve the problem with very little information.

The other is from the customer demand to the product configuration reasoning process, which is a predictive reasoning problem. Typically, mathematical models are built for specific formal design issues. One of the characteristics of this mathematical model is that the solution space can be large and can be analyzed with big data. Therefore, inference methods such as artificial neural networks (ANN) [12, 13], fuzzy inference (FI) [14, 15], rough set theory (RST) [14, 15], and Bayesian networks $(\mathrm{BN})$ ) [16-18] is widely used to present a set of best solutions for customers. S Han et al. [13] applied ANN to the conceptual design of the car, established the ANN model, and predicted the configuration of the concept car. In YS Juang et al. [15] using FI to change the subjective decisionmaking of traditional machine tool plants, the Customer Demand Information System (CRIS) was established. CRIS supports customers and service personnel in providing a systematic way to meet and analyze customer needs. P Zheng et al. [19] proposed a weighted interval rough method, which aims to improve existing methods by explaining objectively and more accurately the various customer demand preferences. In Y Wang et al. [17] using Bayesian factors, a method of quantifying the likelihood of a particular event occurs to predict trends in customer demand. Y Wang et al. [18] discussed the problem of mapping customer requirements to products and built a probability-based naive bias classifier using customer selection data. The classifier takes the new customer demand as input and the output is a product variable that the customer may be satisfied with.

From the above literature we find that different methods have different advantages and disadvantages. Table 1 gives a comparison of the capabilities of BN, FI, RST, and BN, from the ability to obtain customer demand data to the accuracy of the reasoning process.

The comparison shows that $\mathrm{BN}$ has great advantages in dealing with uncertainty and missing data and can be used to solve the uncertainty reasoning of complex systems and data analysis, which is consistent with the inaccuracy and incompleteness of customer demand expression. At the same time, most BN models are a single analysis of customer needs and little research on custom product forecasts. Therefore, using $\mathrm{BN}$ to predict the product configuration that meets customer needs is practical.

In this article, we can predict a satisfactory product mix with incomplete and uncertain customer needs. To achieve this goal, we have established a BN-based customized product model, which mainly includes model nodes and their related information and relationships between nodes and conditional probability distribution tables. Nodes can be divided into three categories: customer information, product features, and product configuration. Based on a customized product model, the customer selects some nodes and their associated information. Companies can accurately predict the final product model through the Node Tree (JT) algorithm [20, 21] and make important and directional texts for customer demand nodes to determine the focus of customer needs and provide a recommendation model for product optimization.

This paper proposes a method based on Bayesian network for custom products and establishes a framework for product preparation methods under incomplete requirements. As shown in Figure 1, first, the enterprise establishes a customized product model, which mainly includes model nodes and the related information of the nodes, the relationship between the nodes, and the conditional probability distribution table between the nodes. Then, the customer selects relevant information of some of the nodes and nodes according to the enterprise customized product model. The company obtains customer choices through an online customization platform and analyzes, infers, and accurately predicts the product model that the customer ultimately expects through the Bayesian network. Finally, for the node inspection of the customer requirements and the optimization of the product model, the node test is divided into importance and directionality. When the customer raises an objection to the role of a node, the system carries out the importance test and feeds the result back to the customer and the enterprise. The customer adjusts his choice according to the situation, and the enterprise optimizes the product model. When the customer's node directionality test conflicts, the system automatically feeds back to the customer. The customer chooses to modify the demand or ignore the demand conflict according to the prompt. The ignored demand conflict is also provided to the enterprise as the product improvement direction.

\section{The Establishment of Customized Product Model}

\subsection{Introduction}

Definition 1. BN is a directed acyclic graph and consists of two parts:

(1) The directed acyclic graph has $\mathrm{N}$ nodes representing random variables. Line segments between nodes represent the relationship between them.

(2) A conditional probability table associated with each node. Since the nodes and their relationships (conditional probability tables) are known, the BN can represent the joint probability distribution of all the nodes therein.

In a generalized model of product configuration methods under incomplete customer requirements, as shown in 
TABLE 1: The ability comparison of BN, FI, RST, and BN.

\begin{tabular}{|c|c|c|c|c|}
\hline \multirow{2}{*}{ Content } & \multicolumn{4}{|c|}{ Method } \\
\hline & ANN & FI & RST & $\mathrm{BN}$ \\
\hline Sample demand & large & small & small & large \\
\hline Influence of prior knowledge & weak & weak & no & strong \\
\hline Logical inference ability & slow & no & fast & middle \\
\hline Handing capacity to the missing data & no & no & no & yes \\
\hline
\end{tabular}

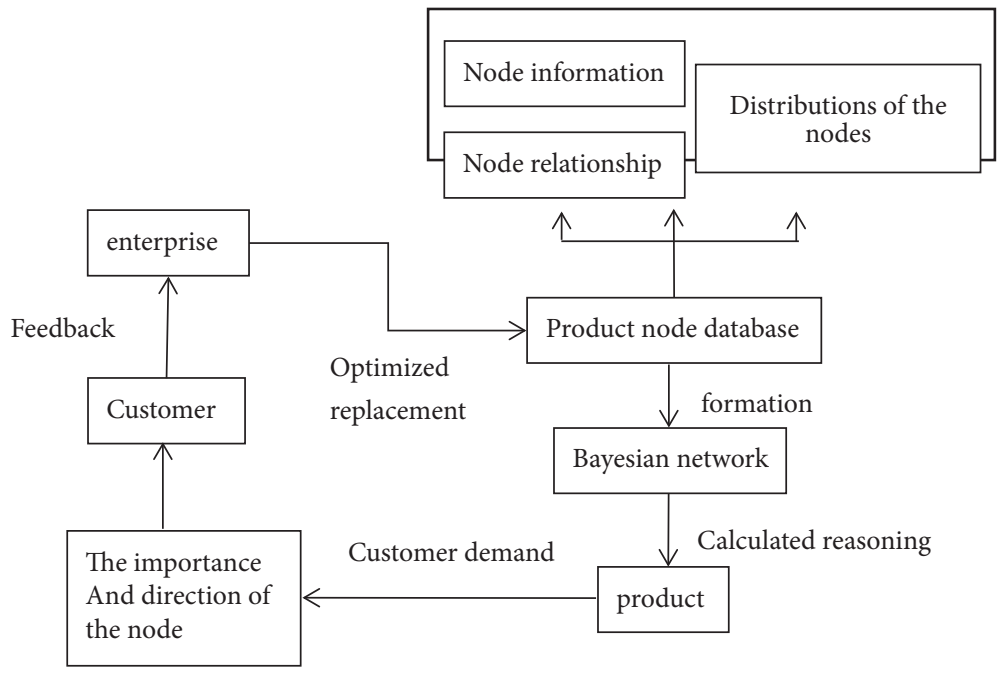

FIgURE 1: Product configuration method under incomplete customer demand.

Figure 1, the most basic and simple structure of Bayesian network "architecture" is a linear, acyclic graph. The nodes in the graph represent random variables between nodes. The connection indicates the relationship between the variables. The network uses Bayesian rules to calculate network propagation. If the probability of the initial node and the conditional probability between all nodes are determined, then the distribution state of all nodes in the network can be quantified.

For two events $\mathrm{Y}$ and $\mathrm{Z}$, the Bayesian rules have the following meanings:

$$
P\left(\frac{Z}{Y}\right)=\frac{P(Y / Z) P(Z)}{P(Y)}
$$

More importantly, if the state of any node in the network is determined, the network itself can use Bayesian rules to perform forward or reverse calculations in the network, thereby deriving the probability that any node in the network will change later. Therefore, the ability to apply this rigorous but easy-to-handle visual model for what-if analysis is the primary reason for using Bayesian networks.

As shown in Figure 2, the selection of node $A$ 's status information will directly affect the probability of the nodes $B$ 's and C's. Thus, we can get the conditional probability distribution table of $B$ and $C$, and because of the continuous effect, a probability network will be formed.

$\mathrm{BN}$ is not only a graphical modeling tool based on Bayesian probability theory, but also has the characteristics of uncertain reasoning ability, which is more prominent in incomplete information prediction. Therefore, it can be used to solve inference prediction problems in the field of product configuration. Due to the specificity of customization, customer requirements and product configuration should be consistent, which can be achieved by establishing a BN model based on a customized product model. In this article, we use customer information, product features, and configurations to form a $\mathrm{BN}$ structure. Let $\mathrm{F}=\{\mathrm{F} 1, \mathrm{~F} 2, \ldots, \mathrm{Fnf}\}$ be the customer information, and let $\mathrm{Q}=\{\mathrm{Q} 1, \mathrm{Q} 2, \ldots, \mathrm{Qnq}\}, \mathrm{C}$ $=\{\mathrm{C} 1, \mathrm{C} 2, \ldots, \mathrm{Cnc}\}$ be the product function and product configuration.

2.2. Structure Establishment. The first step is to select the variables related to customer information, product function, and product configuration, which include node $F n_{f}, Q n_{q}, C n_{c}$ and their related information $x_{f i}, x_{q i}, x_{c i}$. To achieve it, we should

(1) determine the target of the customized product model;

(2) determine the specific node status information related to the product configuration; 


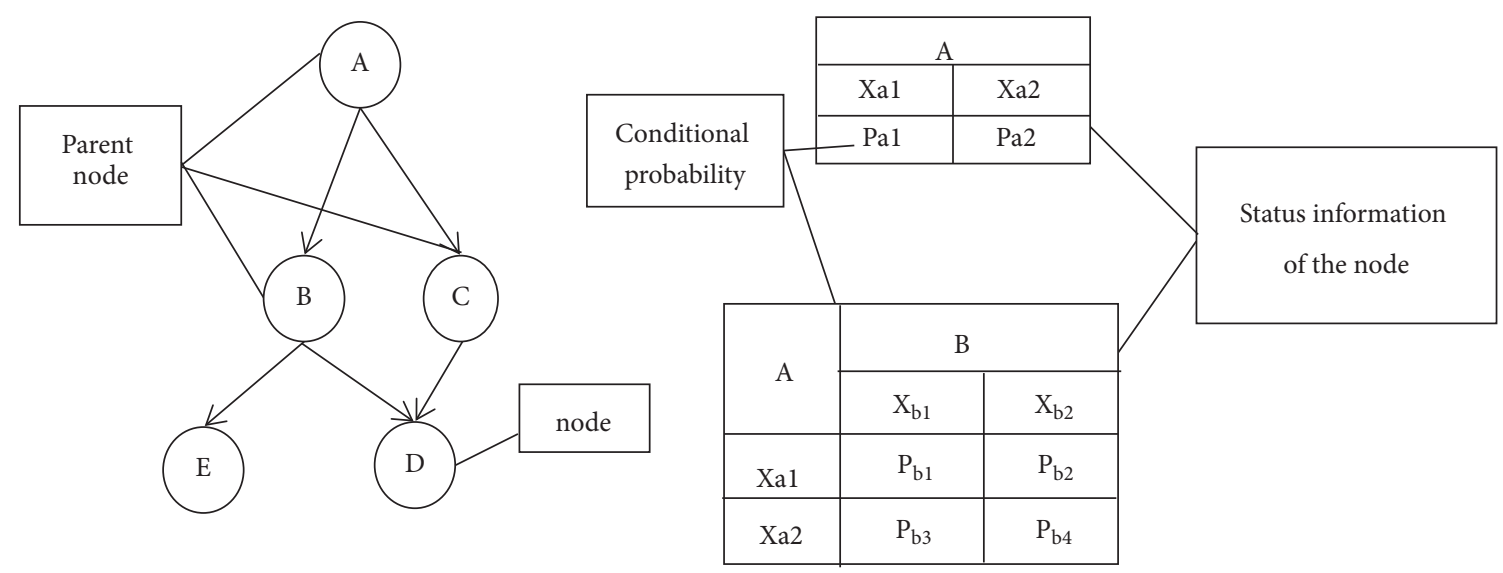

FIGURE 2: Complete BN.

(3) organize this status information into incompatible and finite variables.

For example, in the process of predicting a customized multifunctional nursing bed, the goal of the product is determined to produce a complete multifunctional nursing bed. Customer information includes upper limb dysfunction and lower limb dysfunction. Product function includes side turning stability, and its status information is strong or weak. Product configuration includes bed material.

The second step is to establish a directed acyclic graph, in which each node is independent of others.

As shown in Figure 3, the model structure is a special directed acyclic graph-dendrogram. Nodes $F n_{f}, Q n_{q}, C n_{c}$ are used as the nodes of $\mathrm{BN}$, which can be also be regarded as a customer's preference set. And the directed edges between nodes intuitively express their relationship. For example, in the process of predicting a customized multifunctional nursing bed, upper limb dysfunction and lower limb dysfunction will directly affect the probability of strong and weak stability, which will further affect the selection of bed material.

The inference of $\mathrm{BN}$ whose structure is not a dendrogram is generally NP problem; however, using this special structure-dendrogram has the advantage of greatly speeding up the inference, improving the efficiency of product configuration, and achieving the rapid response to customers.

2.3. Parameter Learning. The third step is the establishment of conditional probability distribution table.

After constructing the BN structure, we need to learn the conditional probability table for each node in the network. Learning is done through statistical data samples in the database, while customer information, product features, and configuration are complete examples of the database. Then we can use statistical methods and bias methods to calculate the conditional probability distribution. The learning process is divided into two steps.

(1) Formatting the Results. Each configuration result is recorded in the format shown in Table 2, where each variable is a Boolean value $\{0,1\}$, and 1 indicates that the customer selects the node; otherwise it is 0 .

For example, in the process of predicting a customized multifunctional nursing bed, Fi = xfi can be "upper limb dysfunction = yes", Qi = xqi can be "safety=low", and Ci = xci can be "push rob=lead screw nut".xfi, xqi, xci are node status information.

(2) Using the Database to Learn the Conditional Probability. Conditional probability learning uses large data statistics. In classical statistics, we generally use the maximum likelihood estimation method (MLE) to study the conditional probability table. The learning of probability is a continuously revised and repetitive process; the prior probability is generally summarized by the expert experience and then modify the prior probability through the calculation with the posterior probability of the last learning becoming a priori probability of the next learning in order to maintain that the parameters can continue learning. The process is shown in Figure 4.

For this problem, the BN toolbox BNT based on Matlab is currently the most versatile software. In this paper, BNT will be used to assist in the construction of conditional probability table.

\section{Inference}

When configuring products based on custom information, the information provided by customers is often ambiguous due to customer knowledge and expressiveness limitations. When a customer provides valid information to one or more nodes of the $\mathrm{BN}$, we calculate complete customer information, product features, and product configurations based on the available information. This process is called $\mathrm{BN}$ inference.

The basic method for calculating the conditional probability is the normalization of marginalization and joint probability. In theory, based on the joint probability of all variables in the network, we can obtain the probability of an arbitrary variable or the low-order joint probability 


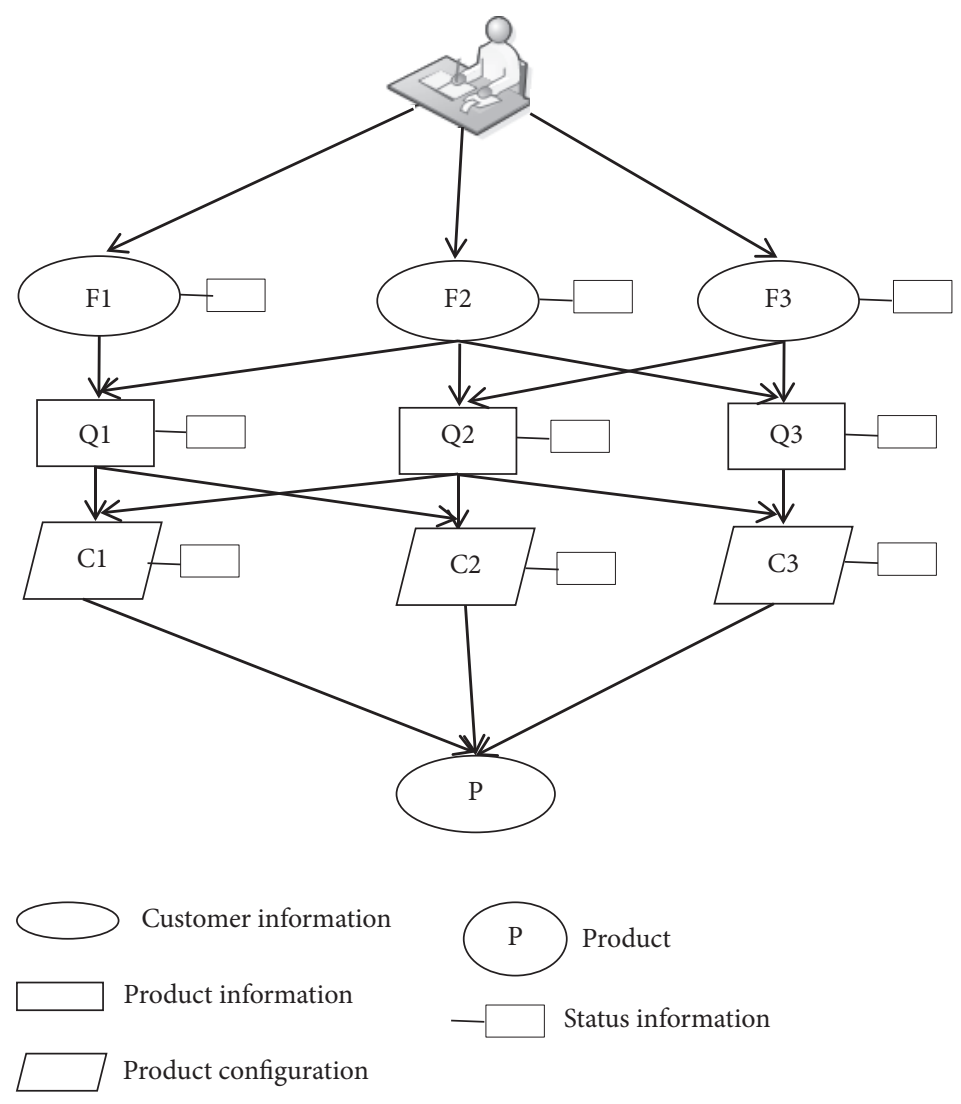

FIGURE 3: BN structure diagram.

TABLE 2: Sample configuration format.

\begin{tabular}{lccc}
\hline Sample number & $F_{i}=x_{f i}$ & $Q_{i}=x_{q i}$ & $C_{i}=x_{c i}$ \\
\hline 1 & 0 & 1 & 1 \\
\hline 2 & 1 & 1 & 0 \\
\hline
\end{tabular}

of multiple variables by the joint operation of the joint probability and normalize them to obtain the conditional probability. In most customer products, the joint probability satisfies certain conditions, which simplifies the calculation and allows it to be specified and calculated.

Because of the strong independence among the nodes of $\mathrm{BN}$, it is possible to express a joint probability according to a conditional probability chain; the general form is

$$
P\left(X_{1}, X_{2}, \ldots, X_{n}\right)=\prod_{i=1}^{n} p\left(X_{i} \mid X_{1}, X_{2}, \ldots, X_{i-1}\right)
$$

We usually refer to it as a chain rule. And, according to the conditional probability formula, $P(A \mid B)=P(A B) / P(B)$; we can get

$$
P\left(X_{i} \mid X_{m}, \ldots, X_{n}\right)=\frac{P\left(X_{i}, X_{m}, \ldots, X_{n}\right)}{P\left(X_{m}, \ldots, X_{n}\right)}
$$

3.1. JT Algorithm. But the customized product model is usually a large set of random variables, specifying all joint probability or lower order joint probability will be a very large task, and the computational complexity will be large; then we can use JT algorithm to solve this problem.

Definition 2. If there is only one path between any two nodes in the $\mathrm{BN}$, the network is called a single-connected network; otherwise it is called a multiconnected network.

This algorithm can not only solve the inference in both the single-connected network and the multiconnected network. Especially, the algorithm is very convenient when there are multiple query nodes in the network.

It uses the JT expression to express the joint probability distributions. The main idea is to transform BN into a JT and then calculate the probability by information dissemination process of JT. The process is shown in Figure 5.

The basic flow of the JT algorithm is as follows.

Step 1. Set up the moral graph (MG) of BN structure. MG connects the nodes in a network that have a common child node but are not directly connected to each other and remove 


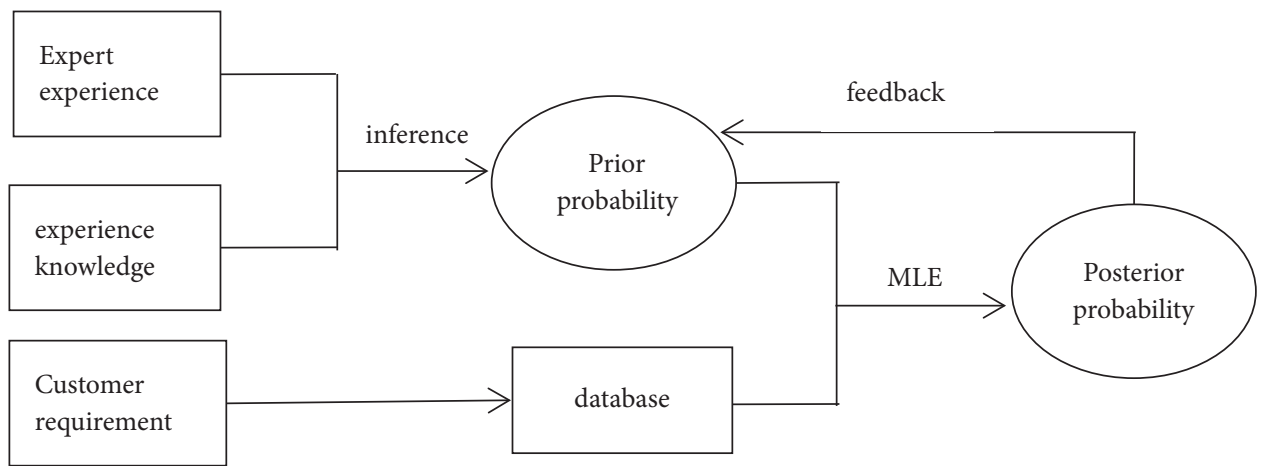

Figure 4: Parameter learning flow chart.

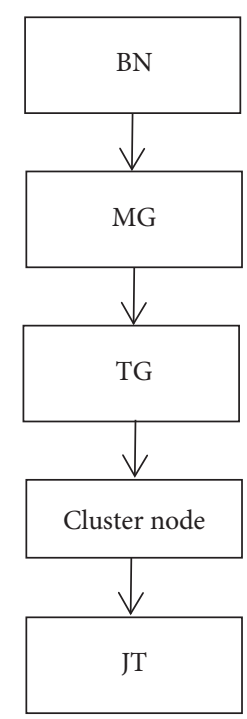

FIgURE 5: The process of constructing a JT.

the direction of each line segment. Figure 5 is a MG of the BN shown in Figure 1.

Step 2. Triangulate MG obtained in Step 1 to obtain a triangulated graph (TG). Add a chord (edge between two nonadjacent nodes) in a cycle that has more than three nodes to triangulate MG. Since MG shown in Figure 6 does not contain the undirected cycle, it is not necessary to triangulate it.

Step 3. Based on Step 2, a set of nodes that are connected to each other is called clique in the undirected graph. A maximum clique is a clique that cannot be contained by other cliques in an undirected graph. Identify all the maximum cliques in the TG and merge the nodes contained in each maximum clique, as a new node, known as the cluster node. The cluster nodes are used as the tree nodes, and then we construct the JT. The separator is the intersection of two cluster nodes. As shown in Figure 6, there are three cluster nodes which are $A B C, B C E$, and $B D$, and disjoint sets are $B$ and $B C$.
Step 4. Establish a JT that must contain all the cluster nodes and separators. Figure 7 is the JT established by the BN of Figure 1.

Step 5. Distribute parameters for each cluster node and separator. Due to the fact that the so-called cluster node and the separator are actually the set of nodes, the parameter here is actually a probability table of all the status of the nodes, usually recorded as $\phi$. At initialization, each element in the table is 1 . Finally, the conditional probability table for each variable in the original network is multiplied by the probability distribution table of the specified cluster node; then we can get the initial given conditional probability.

Step 6. Collect and diffuse the information dissemination process. Suppose that the cluster nodes in the JT are $V$ and $W$; their separator is $S$, and the corresponding probability table is $\phi(V), \phi(W)$, and $\phi(S)$. In the process of disseminate information from $V$ to $W$, we need to update $\phi(S)$ and $\phi(W)$ in accordance with the following method; that is, we have the following. 


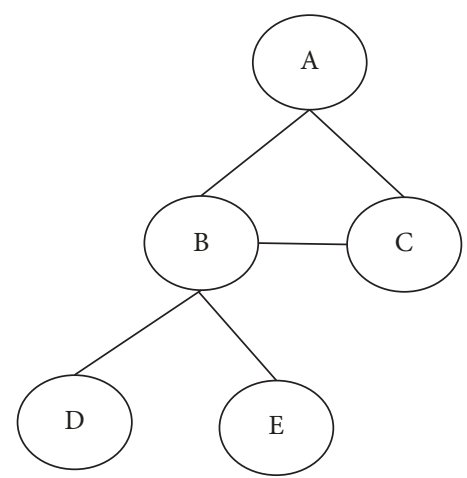

FIgURE 6: The MG of the BN in Figure 1.

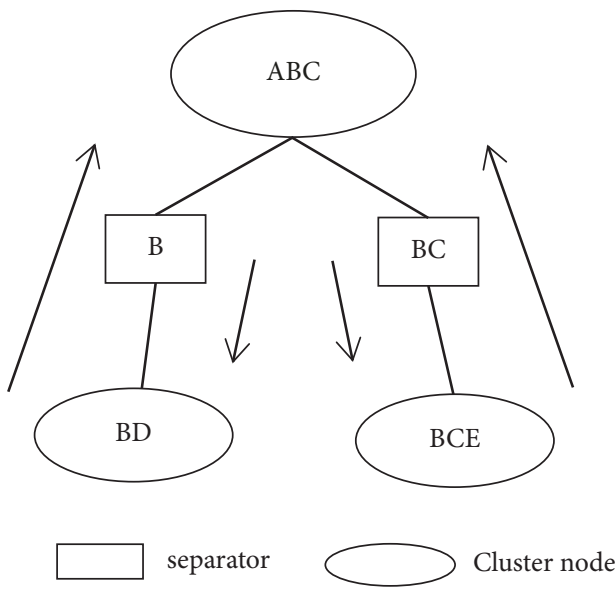

Figure 7: JT constructed by BN in Figure 1.

(1) Separator $S$ absorbs the information:

$$
\phi^{*}(S)=\sum_{V \backslash S} \phi(V)
$$

(2) Update the distribution function of the cluster node $W$ :

$$
\phi^{*}(W)=\phi(W) \frac{\phi^{*}(S)}{\phi(S)}
$$

(3) Update the distribution function of the separator $S$ :

$$
\phi(S)=\phi^{*}(S)
$$

In the JT shown in Figure 6, the cluster node $A B C$ is selected as the root node, the solid arrow indicates the evidence collection process, and the dotted arrow indicates the evidence distribution process.

Step 7. Suppose the query node is Q. After completing the information dissemination, marginalize and normalize the probability table of the cluster node $C$, which is contained in any query node. Then the posterior probability of the query node can be obtained; that is,

$$
\begin{gathered}
P(Q, e)=\sum_{C \backslash Q} \phi(C) \\
P(Q \mid e)=\frac{P(Q, e)}{P(e)}
\end{gathered}
$$

\section{Optimization and Test}

In order to better explain to the customer, make the right and reasonable decision, and continually optimize the product model, we analyze the nodes in $\mathrm{BN}$. BN uses the unified joint probability distribution as the knowledge base, which guarantees the consistency of the knowledge representation and improves the rationality of the inference conclusion. However, this knowledge representation that follows the classical probability theory cannot meet people's thinking habits and automatically generate explanations through inference chain or inference network of direct translation rules. Therefore, the BN interpretation mechanism needs in-depth study.

4.1. Optimization and Importance Test. The customer makes valid information about one or more nodes of BN. The 
nodes used as inference information are called the evidence nodes (evidence for short), such as nodes $\mathrm{Fn}_{\mathrm{f}}, \mathrm{Qn}_{\mathrm{q}}$. The nodes obtained by inference are called the conclusion node (conclusion for short), such as node $C \mathrm{n}_{\mathrm{c}}$. The inference conclusion of $\mathrm{BN}$ is the posterior probability distribution of all nonevidence nodes, but customers tend to care about the posterior probability distribution of one or some specific nonevidence nodes; the enterprise tends to pay attention to the sufficiency and necessity of the evidence node in order to judge whether it should be optimized in the whole model. For example, in the process of predicting a customized multifunctional nursing bed, our focus is the influence of customer information and product function on product configuration. We want to know whether the effect of lower limb dysfunction and safety protection on bed material can be ignored, while the enterprises want to know whether there is an overlap in the influence of lower limb dysfunction and upper limb dysfunction on conclusion nodes, in order to optimize and improve the model.

When a customer examines the effect of a (some) evidence in the model on the inference, it can be removed from the set of evidence (it is treated as a nonevidence in the BN model) and then we should calculate the change of inference conclusion, which is the deletion method. However, since the overlapping phenomenon to inference conclusion may disturb the interpretation, we need to analyze it from the perspective of the necessity and sufficiency.

For a particular nonevidence node $X$ in the product model, its inference conclusion is a posterior probability distribution $P(X \mid E)$ when the evidence set is $E$. Now examine the role of the evidence $L$ in $E$. Deleting $L$ from $E$, we will get a set $E-L$, and its corresponding posterior probability distribution is $P(X \mid E-L)$.

Definition 3. Consider $P(X \mid E)$ and $P(X \mid E-L)$ as vectors and use the one-norm of the vector difference $P(X \mid E)-P(X \mid E-$ $L)$ to measure the difference between them and record it as $M(P(X \mid E), P(X \mid E-L))$; that is,

$$
M\left(P\left(\frac{X}{E}\right), P\left(\frac{X}{E-L}\right)\right)=\sum_{i}\left|p_{i}-q_{i}\right|
$$

where $P\left(X=x_{i} \mid E\right)=p_{i}, P\left(X=x_{i} \mid E-L\right)=q_{i}, i=1,2, \ldots, m$, $m$ is the status number of $X$, and $M(P(X \mid E), P(X \mid E-L))$ is the difference value.

The smaller the difference between $P(X \mid E)$ and $P(X \mid E-$ $L)$, the smaller the effect on the calculation accuracy. Therefore, $M(P(X \mid E), P(X \mid E-L))$ measures the degree of necessity of $L$ to the inference conclusion. Let

$$
\lambda(L, X)=\frac{M(P(X / E), P(X / E-L))}{M(P(X / E), P(X))}
$$

And it is called the necessity factor of $L$ for $x$. It reflects the proportion of the necessity of $L$ in the evidence set $E$.

$\lambda(L, X)$ measures the degree of necessity of the evidence $L$ for forming the inference conclusion. If the value of $\lambda(L, X)$ is larger, it shows the necessity is strong. However, if the value is smaller, it cannot be concluded that the effect of the evidence $\mathrm{L}$ on $P(X \mid E)$ is weak, because the effect of $L$ on inference conclusion may overlap with other evidences in the evidence set.

In order to eliminate the interference caused by the overlapping effect of the evidence in set $E$ on inference conclusion, we further investigate the sufficiency of $L$ to the inference conclusion $P(X \mid E)$, that is, the influence of $L$ alone on $P(X \mid E)$. The inference process is the same as the necessity analysis. If all the evidence other than $L$ is deleted from the evidence set $E$, that is, all the evidence nodes other than $L$ are treated as nonevidence nodes, the posterior probability distribution of $\mathrm{x}$ will change $P(X \mid L)$ from $P(X \mid E)$, and the corresponding necessity factor is $\lambda(E-L, X)$, which is the degree of necessity of evidence $E-L$ for forming the inference conclusion.

Thus, $1-\lambda(E-L, X)$ can be used to measure the contribution of $L$ for the formation of inference conclusion $P(X \mid E)$, which means that the degree of necessity of evidence $L$ for forming the inference conclusion. Let

$$
\mu(L, X)=1-\lambda(E-L, X)
$$

And $\mu(L, X)$ is called $L$ 's sufficiency factor for $X$.

Let threshold $\theta=1 /(|E|+1)$, and use it to evaluate the degree of necessity and sufficiency of $L$, where $|E|$ represents the cardinality of the set $E$ which is the number of verified evidence nodes.

To sum up, according to the rules in Table 3 , the threshold $\theta$ takes the value of $\theta, \lambda(L, X)$, and $\mu(L, X)$ to generate a more detailed explanation, and the evidence can be divided into critical evidence, necessary evidence, sufficient evidence, and general evidence. Customers and enterprises can adjust the selection and optimize the model based on the analysis results.

4.2. The Directivity Test. In the process of customizing product, the directional influence between nodes is also a key point, which is helpful to better analyze the relationship between customer information and product configuration. For example, "upper limb dysfunction = yes" and "safety protection = high" both have the influence on "bed material = stainless steel" in the same direction, and they can be achieved at the same time when we make the choice. If there is a reverse effect, we need to communicate with the customer to explain that the choice of these two nodes cannot be achieved at the same time.

Definition 4. Let $P(X)=\left(p_{1}, p_{2}, \ldots, p_{m}\right), Q(X)=$ $\left(q_{1}, q_{2}, \ldots, q_{m}\right)$ be the probability distribution of nonevidence node $X$ in BN model, $w_{i}=\operatorname{sgn}\left(p_{i}-q_{i}\right)$. The vector $W=$ $\left(w_{1}, w_{2}, \ldots, w_{m}\right)$ is the direction of change from $P(X)$ to $Q(X)$. And let the direction of change from another probability distribution $R(X)$ of $X$ to $Q(X)$ be $V=\left(v_{1}, v_{2}, \ldots, v_{m}\right)$. If the number of $w_{i}$ 's and $v_{i}(i=1,2, \ldots, m)$ 's signs differ less than $[m / 2]$, we call $P(X)$ and $R(X)$ basically have the same direction about $Q(X)$; otherwise $P(X)$ and $R(X)$ basically have the opposite direction about $Q(X)$.

For the evidence $L$ in $E$, if the probability distribution $P(X \mid E)$ and $P(X \mid L)$ basically have the same direction about 
TABLE 3: The effect of evidence on inference conclusion.

\begin{tabular}{|c|c|c|c|c|}
\hline$\lambda(L, X)>\theta$ & $\mu(L, X)>\theta$ & $\begin{array}{l}L \text { plays an important role in } \\
\text { the conclusion and can't be } \\
\text { replaced by other evidence } \\
\text { in } E\end{array}$ & critical evidence & $\begin{array}{l}\text { Customers don't have a } \\
\text { greater choice of results. } \\
\text { The company should keep } \\
\text { the node and choose the } \\
\text { other node to optimize the } \\
\text { model }\end{array}$ \\
\hline$\lambda(L, X)>\theta$ & $\mu(L, X)<\theta$ & $\begin{array}{l}L \text { contributes little to the } \\
\text { conclusion, but is } \\
\text { indispensable in } E \text {. }\end{array}$ & necessary evidence & $\begin{array}{l}\text { Customers don't have a } \\
\text { greater choice of results. } \\
\text { The company should keep } \\
\text { the node and choose the } \\
\text { other node to optimize the } \\
\text { model }\end{array}$ \\
\hline$\lambda(L, X)<\theta$ & $\mu(L, X)>\theta$ & $\begin{array}{l}L \text { plays an important role in } \\
\text { the conclusion, but it can } \\
\text { be replaced by others } \\
\text { because of overlapping. }\end{array}$ & sufficient evidence & $\begin{array}{l}\text { Customers don't have a } \\
\text { greater choice of results. } \\
\text { The company can remove it } \\
\text { for optimization. }\end{array}$ \\
\hline$\lambda(L, X)<\theta$ & $\mu(L, X)<\theta$ & $\begin{array}{l}L \text { contributes little to the } \\
\text { conclusion, and it can be } \\
\text { replaced by others because } \\
\text { of overlapping. }\end{array}$ & general evidence & $\begin{array}{l}\text { Customers don't have a } \\
\text { greater choice of results. } \\
\text { The company can remove it } \\
\text { for optimization. }\end{array}$ \\
\hline
\end{tabular}

$P(X), L$ will support the establishment of the inference conclusion, and the choice of the customer has no conflict with the result of the configuration. On the other hand, if the probability distribution $P(X \mid E)$ and $P(X \mid L)$ basically have the opposite direction about $P(X), L$ will weaken the establishment of the inference conclusion and the choice of the customer and the configuration will conflict. And if the sign is +1 , then the evidence $L$ will promote the choice of the node and vice versa. Let $E^{+}$be a set of all the positive evidence in $E$ and $E^{-}$be a set of all the reverse evidence in $E$. If $E^{+}$is equal to $E^{-}$, we can say all the evidence in $E$ is consistent; otherwise it shows that there is a conflict phenomenon of evidences in $E$. According to $E^{+}$and $E^{-}$, we can list the evidence that support or oppose the conclusion to explain the conflict between the evidence.

\section{Case Study}

5.1. Model Establishment. In order to evaluate the effectiveness of $\mathrm{BN}$ on product configuration, a multifunctional nursing bed is used as an example. Multifunctional nursing bed is designed for long-term bedridden patients, using the unique structure of double fold surface which can be adjusted into a flat state, a horizontal state, and other state spaces, and it also has the device for helping patients to get up and turn over, which enhances the level of nurse, improves the patients' living quality, and solves a series of difficult problems in nursing. Multifunctional nursing bed is already not the exclusive product of the hospital, and now the market also produced home style nursing beds which gradually go into the family and play a great role in the family care. Because of the customers' incomplete understanding of the function and structure about the multifunctional nursing bed, their expression cannot effectively help the enterprise to make decision. Therefore, it is a good choice to use $\mathrm{BN}$ for inference and prediction.
According to the market investigation, we need to clarify the structure and function of the multifunctional nursing bed and understand its status information and then build the BN structure model as shown in Figure 8.

$\mathrm{BN}$ is a directed graphical model used to represent the probability of connection between variables. It provides a natural means of representing causal information to discover potential relationships between data. BN uses nodes to represent variables and directed edges to represent dependencies between variables. Its detailed description is as follows:

(1) A set of random variables form a network node: variables can be discrete or continuous.

(2) A set of connected edges or arrows forming a set of edges: if there is a directed edge from node $\mathrm{X}$ to node $\mathrm{Y}$, then $\mathrm{X}$ is said to be a parent of $\mathrm{Y}$.

(3) Each node $\mathrm{X} i$ has a conditional probability distribution $\mathrm{P}(\mathrm{X}$ i |Parents(X i )), which indicates the influence of its parent node on the node.

(4) There is no directed ring in the figure, so it is a directed acyclic graph.

In this paper, a tree-shaped Bayesian network is adopted. The Bayesian network has strong uncertainty reasoning ability, and the prediction effect on incomplete information is more prominent. Taking the multifunctional nursing bed as an example, the product configuration problem driven by customer demand is solved. According to the particularity of customer customization, in order to meet the customer's needs and product configuration consistency, this paper directly uses customer information, product function, and product configuration as a Bayesian network structure.

The enterprise establishes a model of customized products. The model mainly includes related information between model nodes and nodes, the relationship between nodes, and the conditional probability distribution between nodes. The customer selects the nodes according to the customized product model, and the enterprise customizes through online. 


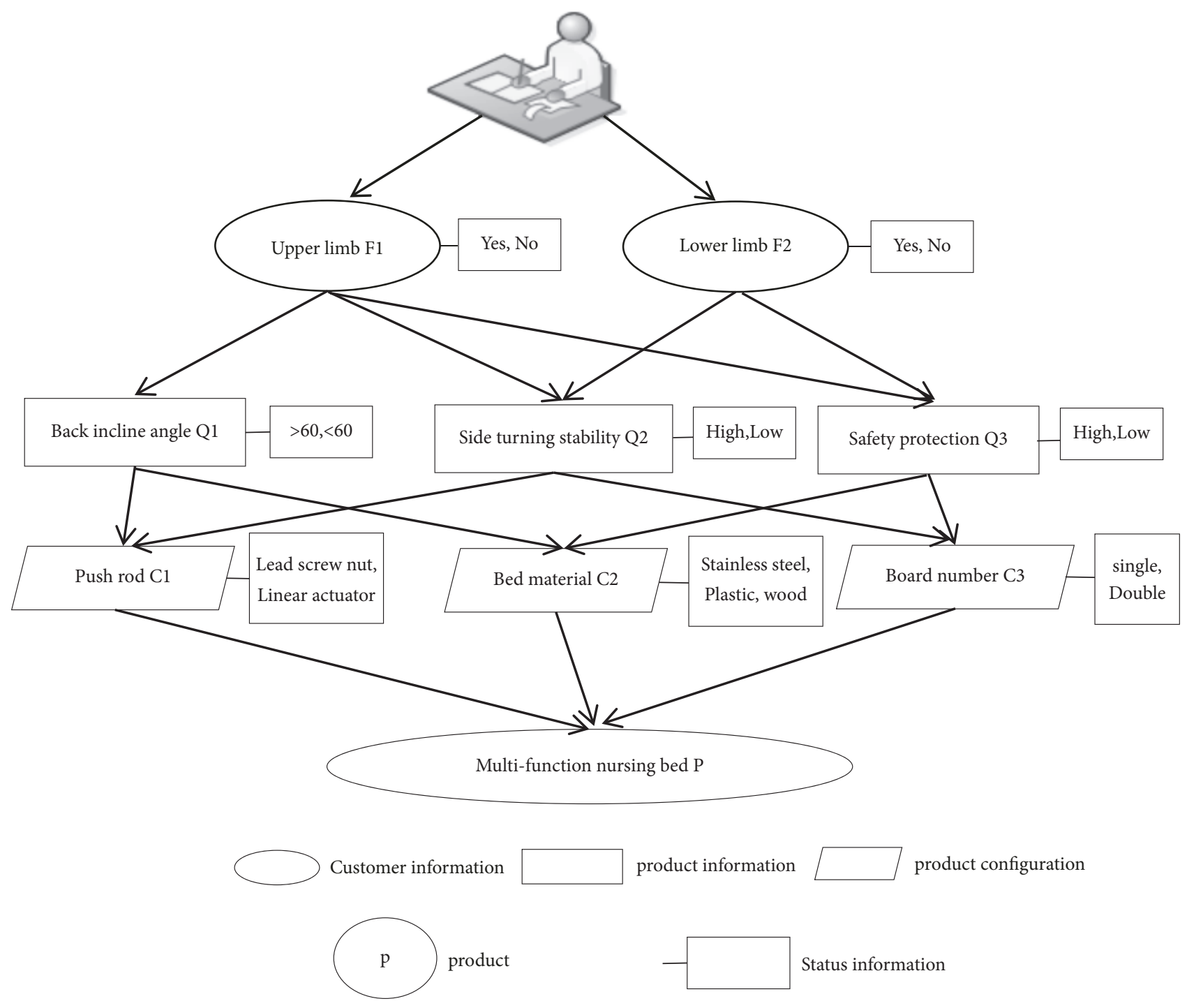

FIGURE 8: BN structure of multifunctional nursing bed.

The platform obtains the customer choice. These nodes also serve as the user's preference set. The directed edge directly represents the relationship between the nodes. This paper applies BNT to establish the user's BN model and first creates the BN structure. The conditional probability of a network node is to learn the data sample. Each field in the record is a node in the logical structure of the product. There are two kinds of selection behaviors for the users of these nodes; 0 means that this function is not selected, and 1 means selection. So whether each node is selected to use 0 or 1 to confirm. After learning the structure through BNT, as shown in Figure 8 , there are 8 nodes, and the value of each node variable is $\{0,1\}$. The directed edge reflects the relationship between the nodes.

The advantage of being able to use this tree-shaped Bayesian network structure is that it can greatly improve the speed of reasoning and improve the efficiency of product configuration when reasoning. Many inference algorithms can be completed in linear time, achieving rapid response to customers. At present, the research on $\mathrm{BN}$ is mainly reasoning and learning. The reasoning of BNT is the process of applying $\mathrm{BN}$ to solve the problem including accurate reasoning and approximate reasoning as shown in Figure 9.

It consists of a directed acyclic graph and eight marginal or conditional probability tables. The variable names are customer information set upper limb dysfunction $F_{1}$, lower limb dysfunction $F_{2}$, function set back incline angle $Q_{1}$, side turning stability $Q_{2}$, safety protection $\left.Q_{3}\right\}$, and product configuration set \{push rod type $C_{1}$, bed material $C_{2}$, Board number $\left.C_{3}\right\}$. The corresponding choices for each variables are $\{$ Yes, No $\}$ for $F_{1}$, $\{$ Yes, No $\}$ for $F_{2},\left\{>60^{\circ}, \leq 60^{\circ}\right\}$ for $Q_{1}$, $\{$ High,Low $\}$ for $Q_{2},\{$ High, Low $\}$ for $Q_{3}$, \{Lead screw nut, Linear actuator $\}$ for $C_{1}$, \{Stainless steel, Plastic, Wood $\}$ for $C_{2}$, and $\{$ Single, Double $\}$ for $C_{3}$.

It is a complex process to estimate the parameters of $\mathrm{BN}$. In this paper, we use the parameter estimation method in classical statistics to solve the problem. In general, we use MLE to study conditional probability tables, and The BN 


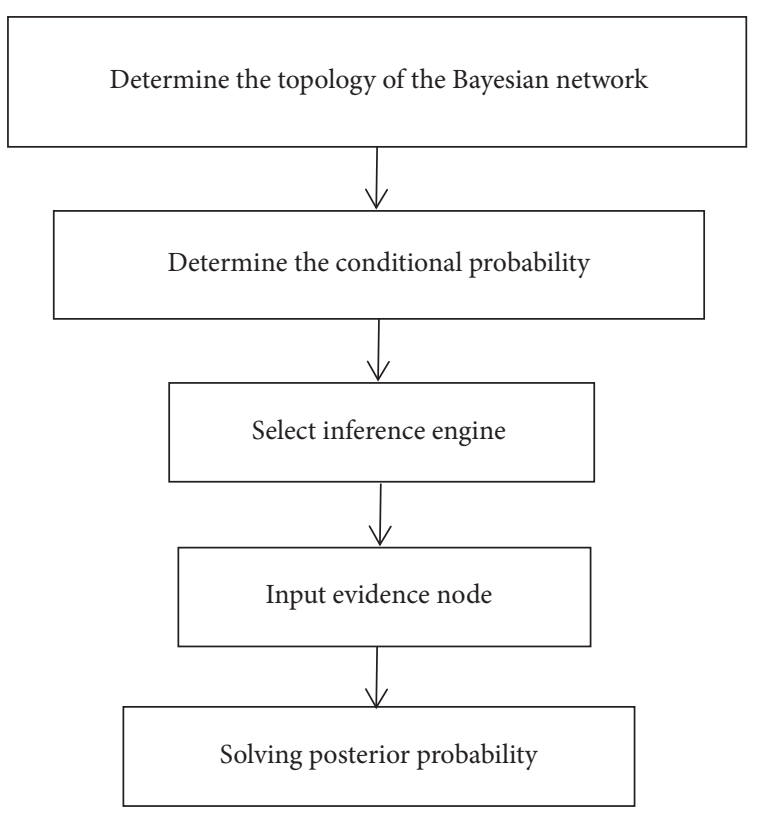

FIGURE 9: Reasoning process of BNT.

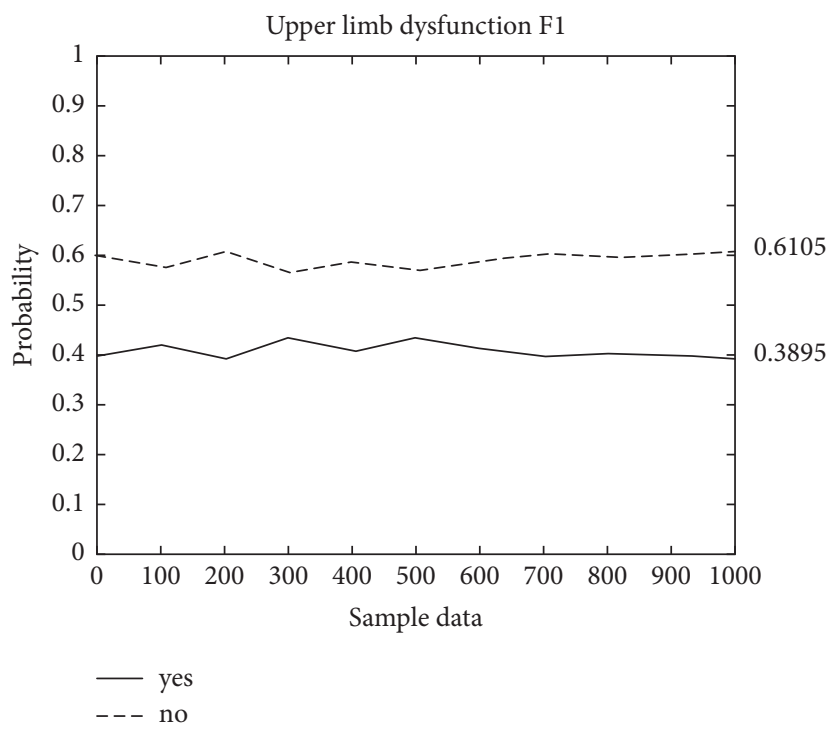

FIGURE 10: The parameter learning process of $F_{1}$.

toolkit BNT is used to assist learning. Then we can construct conditional probability tables. The sample data used for the training parameter model are obtained from the network big data and nursing bed company sales data.

1000 customers are selected as sample source. The customers select the final product according to individual circumstances. The process is learned with BNT in Matlab. The results of one of nodes are shown in Figure 10, which shows the learning process of upper limb dysfunction $F_{1}$.

The remaining conditional probability tables obtained by the learning results are shown in Table 4.

We have obtained a complete BN model.
5.2. Product Configuration Inference. We get a complete $\mathrm{BN}$ model of multifunctional nursing bed. Because of the incomplete expression or lack of experience, usually customers can only make an explicitly choice for some nodes in the 8 nodes. Of course, the more nodes the customer defines, the more accurate the reasoning is. For convenience, we assume that the customer can make a choice for only two nodes, namely, upper limb dysfunction $F_{1}=$ "yes" and lower limb dysfunction $F_{2}=$ "yes".

Now configure and inference product based on the product configuration is set to verify the correctness and effectiveness of the model. 
By the independence of the network structure, we can get the following relations:

$$
\begin{aligned}
& P\left(F_{1}, F_{2}\right)=P\left(F_{1}\right) P\left(F_{2}\right) \\
& P\left(Q_{1} \mid F_{1}, F_{2}\right)=P\left(Q_{1} \mid F_{1}\right) \\
& P\left(Q_{2} \mid F_{1}, F_{2}, Q_{1}\right)=P\left(Q_{2} \mid F_{1}, F_{2}\right) \\
& P\left(Q_{3} \mid F_{1}, F_{2}, Q_{1}, Q_{2}\right)=P\left(Q_{3} \mid F_{1}, F_{2}\right) \\
& P\left(C_{1} \mid F_{1}, F_{2}, Q_{1}, Q_{2}, Q_{3}\right)=P\left(C_{1} \mid Q_{1}, Q_{2}\right) \\
& P\left(C_{2} \mid F_{1}, F_{2}, Q_{1}, Q_{2}, Q_{3}, C_{1}\right)=P\left(C_{2} \mid Q_{1}, Q_{3}\right) \\
& P\left(C_{3} \mid F_{1}, F_{2}, Q_{1}, Q_{2}, Q_{3}, C_{1}, C_{2}\right)=P\left(C_{3} \mid Q_{2}\right) \\
& P\left(C_{1}, F_{1}=\text { "yes", } F_{2}=\text { “yes" }\right)=\sum_{\mathrm{Q}_{1}, \mathrm{Q}_{2}, \mathrm{Q}_{3}, C_{2}, \mathrm{C}_{3}} P\left(F_{1}=\text { “yes”, } F_{2}=\text { "yes", } Q_{1}, Q_{2}, Q_{3}, C_{1}, C_{2}, C_{3}\right)
\end{aligned}
$$

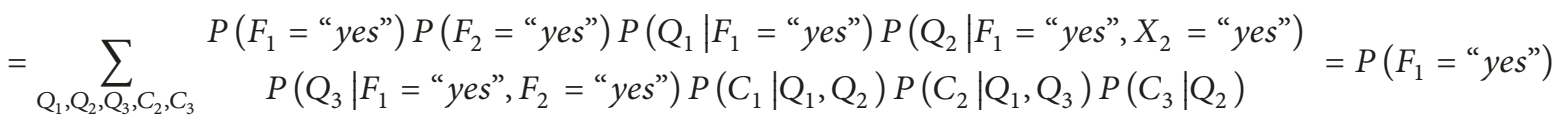

$$
\begin{aligned}
& \cdot P\left(F_{2}=\text { “yes” }\right) \sum_{Q_{1}} P\left(Q_{1} \mid F_{1}=\text { “yes" }\right) \sum_{Q_{2}} P\left(Q_{2} \mid F_{1}=\text { “yes”, } F_{2}=\text { “yes” }\right) \sum_{Q_{3}} P\left(Q_{3} \mid F_{1}=\text { "yes”, } F_{2}=\text { “yes" }\right) \\
& \cdot P\left(C_{1} \mid Q_{1}, Q_{2}\right) \sum_{C_{2}} P\left(C_{2} \mid Q_{1}, Q_{3}\right) \sum_{C_{3}} P\left(C_{3} \mid Q_{2}\right) \\
& P\left(F_{1}, F_{2}, Q_{1}, Q_{2}, Q_{3}, C_{1}, C_{2}, C_{3}\right)=P\left(F_{1}\right) P\left(F_{2}\right) \\
& \cdot P\left(Q_{1} \mid F_{1}\right) P\left(Q_{2} \mid F_{1}, F_{2}\right) P\left(Q_{3} \mid F_{1}, F_{2}\right) \\
& \cdot P\left(C_{1} \mid Q_{1}, Q_{2}\right) P\left(C_{2} \mid Q_{1}, Q_{3}\right) P\left(C_{3} \mid Q_{2}\right)
\end{aligned}
$$

According to the probability distribution, it can be obtained from formula (2),
In the calculation process, $P\left(Q_{3} \mid F_{1}, F_{2}\right), P\left(C_{2} \mid Q_{1}, Q_{3}\right)$, and $P\left(C_{3} \mid Q_{2}\right)$ are eliminated from the product by the normalization. When taking into $C_{1}$ 's value, the marginal probability, and conditional probability, we can get the value of $P\left(C_{1}, F_{1}=\right.$ "yes", $F_{2}=$ "yes").Then, according to the formula (3), we can get the value of $P\left(C_{1} \mid F_{1}=\right.$ "yes", $F_{2}=$ "yes" $)=P\left(C_{1}, F_{1}=\right.$ "yes", $F_{2}=$ "yes" $) / P\left(F_{1}=\right.$ "yes", $F_{2}=$ "yes" $)$.

The results are shown in Table 5 , in which $P\left(C_{1}\right)$ is obtained from the sample data statistics.

Since the model belongs to the multiconnected $\mathrm{BN}$ model, it can also be inferred by the JT algorithm. Based on the above method, the JT of BN about the multifunctional nursing bed is shown in Figure 11.

And according to formula (4) to formula (8), we get the probability distribution of bed material $C_{2}$ and Board number $C_{3}$ that is shown in Tables 6 and 7.

Therefore, the product configuration of the maximum probability is "linear actuator, stainless steel, double board". The several results that have greater probability are as follows: product A\{linear actuator, stainless steel, double board\}, product $B\{$ linear actuator, stainless steel, single board $\}$, product C $\{$ linear actuator, plastic, double board $\}$, product $\mathrm{D}\{$ linear actuator, plastic, single board \}, and product E\{lead screw nut, stainless steel, double board\}.

As shown in Figure 12, the results of $\mathrm{BN}$ are basically consistent with that of the market access. ANN is prone to errors in the face of incomplete customer information, but 
TABLE 4: Conditional probability distribution table.

\begin{tabular}{|c|c|c|c|c|c|}
\hline \multicolumn{2}{|c|}{ Upper limb dysfunction $F_{1}$} & \multicolumn{4}{|c|}{ Lower limb dysfunction $F_{2}$} \\
\hline yes & 0.3895 & & & & \\
\hline no & 0.6105 & & & & \\
\hline \multirow{2}{*}{\multicolumn{2}{|c|}{ Upper limb dysfunction $F_{1}$}} & \multicolumn{4}{|c|}{ Back incline angle $Q_{1}$} \\
\hline & & \multicolumn{2}{|c|}{$>60^{\circ}$} & \multicolumn{2}{|c|}{$\leq 60^{\circ}$} \\
\hline yes & & \multicolumn{2}{|c|}{0.7563} & \multicolumn{2}{|c|}{0.2437} \\
\hline no & & \multicolumn{2}{|c|}{0.3478} & \multicolumn{2}{|c|}{0.6522} \\
\hline \multirow{2}{*}{ Upper limb dysfunction $F_{1}$} & \multirow{2}{*}{ Lower limb dysfunction $F_{2}$} & \multicolumn{2}{|c|}{ Side turning stability $Q_{2}$} & \multicolumn{2}{|c|}{ Safety protection $Q_{3}$} \\
\hline & & high & low & high & low \\
\hline yes & yes & 0.8653 & 0.1347 & 0.9455 & 0.0545 \\
\hline yes & no & 0.4563 & 0.5437 & 0.3965 & 0.6035 \\
\hline no & yes & 0.5854 & 0.4146 & 0.5543 & 0.4457 \\
\hline no & no & 0.2562 & 0.7438 & 0.1983 & 0.8017 \\
\hline \multirow{2}{*}{ Back incline angle $Q_{1}$} & \multirow{2}{*}{ Side turning stability $Q_{2}$} & \multicolumn{4}{|c|}{ Push rod $C_{1}$} \\
\hline & & Lead s & & & uator \\
\hline$>60^{\circ}$ & high & & & & \\
\hline$>60^{\circ}$ & low & & & & \\
\hline$\leq 60^{\circ}$ & high & & & & \\
\hline$\leq 60^{\circ}$ & low & & & & \\
\hline \multirow{2}{*}{ Back incline angle $Q_{1}$} & \multirow{2}{*}{ Safety protection $Q_{3}$} & \multicolumn{4}{|c|}{ Bed material $C_{2}$} \\
\hline & & Stainless steel & Plastic & & \\
\hline$>60^{\circ}$ & high & 0.7332 & 0.2253 & & \\
\hline$>60^{\circ}$ & low & 0.4832 & 0.3432 & & \\
\hline$\leq 60^{\circ}$ & high & 0.4154 & 0.3541 & & \\
\hline$\leq 60^{\circ}$ & low & 0.2478 & 0.4572 & & \\
\hline \multirow{2}{*}{\multicolumn{2}{|c|}{ Side turning stability $Q_{2}$}} & \multicolumn{4}{|c|}{ Board number $C_{3}$} \\
\hline & & & & \multicolumn{2}{|c|}{ double } \\
\hline \multicolumn{2}{|c|}{ high } & \multicolumn{2}{|c|}{0.3745} & & \\
\hline \multicolumn{2}{|c|}{ low } & \multicolumn{2}{|c|}{0.6543} & \multicolumn{2}{|c|}{0.3457} \\
\hline
\end{tabular}

TABLE 5: The inference conclusions of $C_{1}$.

\begin{tabular}{lcc}
\hline Push rod $C_{1}$ & Lead screw nut & Linear actuator \\
\hline$P\left(C_{1}, F_{1}=\right.$ “yes”, $F_{2}=$ “yes") & 0.03165 & 0.24801 \\
\hline$P\left(C_{1} \mid F_{1}=\right.$ “yes”, $F_{2}=$ “yes") & 0.11317 & 0.88683 \\
\hline$P\left(C_{1}\right)$ & 0.2879 & 0.7121 \\
\hline
\end{tabular}

TABle 6: The inference conclusions of $C_{2}$.

\begin{tabular}{lccc}
\hline Bed material $C_{2}$ & stainless steel & plastic & wood \\
\hline$P\left(C_{2}, F_{1}=\right.$ "yes", $F_{2}=$ "yes") & 0.17988 & 0.08881 & 0.01097 \\
\hline$P\left(C_{2} \mid F_{1}=\right.$ "yes", $F_{2}=$ “yes") & 0.64321 & 0.31757 & 0.03922 \\
\hline$P\left(C_{2}\right)$ & 0.4699 & 0.3450 & 0.1851 \\
\hline
\end{tabular}

TABLE 7: The inference conclusions of $C_{3}$.

\begin{tabular}{lcc}
\hline Board number $C_{3}$ & Single board & Double board \\
\hline$P\left(C_{3}, F_{1}=\right.$ "yes", $F_{2}=$ "yes") & 0.11527 & 0.16439 \\
\hline$P\left(C_{3} \mid F_{1}=\right.$ "yes", $F_{2}=$ "yes" $)$ & 0.41219 & 0.58781 \\
\hline$P\left(C_{3}\right)$ & 0.5144 & 0.4856 \\
\hline
\end{tabular}




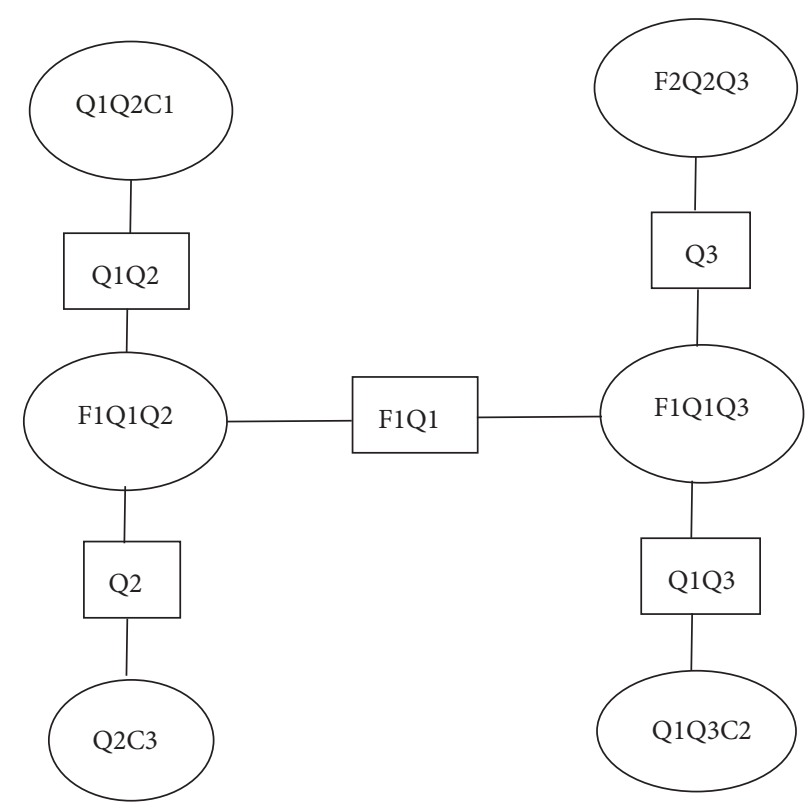

separator

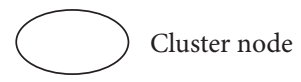

FIGURE 11: The JT of multifunctional nursing bed.

TABLE 8: The inference conclusions of $C_{1}$ in different evidence.

\begin{tabular}{lcc}
\hline Push rod $C_{1}$ & Lead screw nut & Linear actuator \\
\hline$P\left(C_{1}, L\right)$ & 0.15558 & 0.56242 \\
\hline$P\left(C_{1} \mid L\right)$ & 0.21669 & 0.78331 \\
\hline$P\left(C_{1}\right)$ & 0.2879 & 0.7121 \\
\hline$P\left(C_{1}, E-L\right)$ & 0.02932 & 0.08052 \\
\hline$P\left(C_{1} \mid E-L\right)$ & 0.26692 & 0.73308 \\
\hline
\end{tabular}

The difference value can be obtained according to Definition 3 and formula (9). According to formula (10) and formula (11), we can calculate $\lambda\left(L, C_{1}\right)=M\left(P\left(C_{1} \mid E\right), P\left(C_{1} \mid E-\right.\right.$ $L)) / M\left(P\left(C_{1} \mid E\right), P\left(C_{1}\right)\right), \mu\left(L, C_{1}\right)=1-\lambda\left(E-L, C_{1}\right)$. Similarly, other results are shown in Table 9.

Let threshold $\theta=1 /(|E|+1)=1 / 2$. According to Table 3 , lower limb dysfunction $F_{2}=$ "yes" is the necessary evidence.

So lower limb dysfunction $F_{2}=$ "yes" has little effect on push rod type $C_{1}$, but cannot be replaced by other evidence with upper limb dysfunction $F_{1}=$ "yes" acting together on push rod type $C_{1}$; that is, the customer can have a greater choice about the push rod type. The enterprise should reserve lower limb dysfunction $F_{2}$ and select another node to optimize the model.

(2) The Directivity Test. We can calculate the direction from each evidence in $E=\left\{F_{1}=\right.$ "yes", $F_{2}=$ "yes" $\}$ to the final inference conclusion. According to Definition 4, the calculations are shown in Table 10.
It can be seen that the evidence $F_{2}=$ "yes" is positive evidence, and it plays a great role in promoting the choice of "linear actuator". At the same time, $E^{+}$is equal to $E$. That means all the evidence in $E$ is consistent; that is, there is no conflict in the evidence set, and customer requirements can be fully satisfied.

\section{Conclusion}

This paper studies how to make use of the customer's requirement information for product configuration. In the past, the process relied heavily on expert experience. But now a large amount of data that can be easily obtained and algorithm innovation provide a new opportunity for product customization. However, the incompleteness and inaccuracy of customer expressions often affect the accuracy of the results. To solve this problem, this paper presents a customized product model based on BN. The customer selects some nodes and their relevant information according 


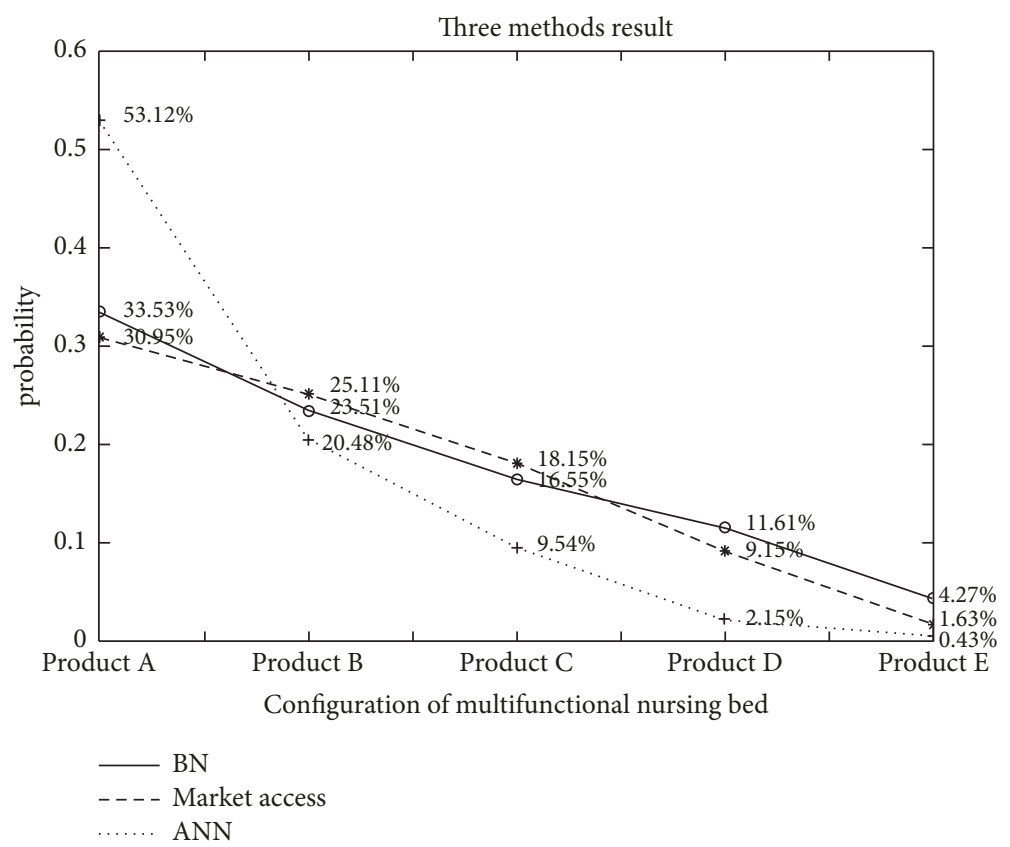

FIGURE 12: Three methods result.

TABLE 9: Interaction factor of $\mathrm{L}$ on $P\left(C_{1}\right)$.

\begin{tabular}{lcccccc}
\hline & \multicolumn{2}{c}{ The probability distribution of $P\left(C_{1}\right)$} & & one-norm & & Interaction factor \\
& Lead screw nut & Linear actuator & & & \\
\hline$P\left(C_{1} \mid E\right)$ & 0.11317 & 0.88683 & $M\left(P\left(C_{1} \mid E\right), P\left(C_{1}\right)\right)$ & 0.34946 & $\mu\left(L, C_{1}\right)$ & $40.75 \%$ \\
\hline$P\left(C_{1}\right)$ & 0.2879 & 0.7121 & $M\left(P\left(C_{1} \mid E\right), P\left(C_{1} \mid E-L\right)\right)$ & 0.3075 & $\lambda\left(L, C_{1}\right)$ & $88.01 \%$ \\
\hline$P\left(C_{1} \mid E-L\right)$ & 0.26692 & 0.73308 & $M\left(P\left(C_{1} \mid E\right), P\left(C_{1} \mid L\right)\right)$ & 0.20704 & $\lambda\left(E-L, C_{1}\right)$ & $59.25 \%$ \\
\hline$P\left(C_{1} \mid L\right)$ & 0.21669 & 0.78331 & & &
\end{tabular}

TABLE 10: The direction from each evidence to the inference conclusion.

\begin{tabular}{|c|c|c|c|c|}
\hline & \multicolumn{2}{|c|}{ The probability distribution and direction of $P\left(C_{1}\right)$} & \multirow{2}{*}{ The number of contrary sign } & \multirow{2}{*}{ Interaction direction } \\
\hline & Lead screw nut & Linear actuator & & \\
\hline$P\left(C_{1}\right)$ & 0.2879 & 0.7121 & & \\
\hline$P\left(C_{1} \mid E\right) / w_{i}$ & $0.11317 /-1$ & $0.88683 /+1$ & & \\
\hline$P\left(C_{1} \mid X_{1}=\right.$ "yes" $) / w_{i}$ & $0.26692 /-1$ & $0.73308 /+1$ & 0 & basically same direction \\
\hline$P\left(C_{1} \mid X_{2}=\right.$ "yes" $) / w_{i}$ & $0.21669 /-1$ & $0.78331 /+1$ & 0 & basically same direction \\
\hline
\end{tabular}

to the model. And through reasoning and calculation with JT algorithm, it recommends the most acceptable product configuration. By using this method, designers can better understand preferences and requirements of customer and test customer demand nodes to identify the focus of customer demand and optimize the product model. This method has higher efficiency and saves more manpower than market access. Compared with ANN, it improves accuracy and makes up the defect of inaccurate prediction based on incomplete information. The main work includes the followings:

(i) Given that customer needs are often expressed in vague language that includes both uncertain and inaccurate information, the bias network is better suited to quantifying uncertainty of customer requirement.
According to the customer's expression of any node in the product model, it can recommend the product configuration that the customer is most likely to accept.

(ii) In the proposed approach, customer demand flexibility is considered. The customer's final choice may differ from what they have expressed in the requirements list. Enterprises can check the nodes to identify the focus of customer demand and better provide reliable advice to customers. At the same time, for the other requirements of the customer, the enterprise can also establish corresponding nodes to analyze whether there is need to optimize the model. 
Further avenues of research might consider improving the efficiency of Bayesian network construction and optimization, especially how to estimate parameters with less training data.

\section{Data Availability}

The data in this article was obtained from the cooperating companies. Data is kept confidential.

\section{Conflicts of Interest}

The authors declared no potential conflicts of interest with respect to the research, authorship, and/or publication of this article.

\section{Acknowledgments}

This research is supported by the National Natural Science Foundation of China (no. 51775156), and the China Scholarship Council (no. 201706695019).

\section{References}

[1] L. L. Zhang, "Product configuration: a review of the state-ofthe-art and future research," International Journal of Production Research, vol. 52, no. 21, pp. 6381-6398, 2014.

[2] G. Oddsson and K. R. Ladeby, "From a literature review of product configuration definitions to a reference framework," AI EDAM Artificial Intelligence for Engineering Design, Analysis and Manufacturing, vol. 28, pp. 413-428, 2014.

[3] M. Aldanondo and E. Vareilles, "Configuration for mass customization: how to extend product configuration towards requirements and process configuration," Journal of Intelligent Manufacturing, vol. 19, no. 5, pp. 521-535, 2008.

[4] Q. Yang, H. Jiao, F. Song, G. Pan, and D. Wei, "Customer requirement acquisition system and requirement expression guidance based on ant colony optimization," Advances in Mechanical Engineering, vol. 9, pp. 1-9, 2017.

[5] C. Chen and L. Wang, "Integrating rough set clustering and grey model to analyse dynamic customer requirements," Proceedings of the Institution of Mechanical Engineers, Part B: Journal of Engineering Manufacture, vol. 222, no. 2, pp. 319-332, 2008.

[6] H.-H. Wu, A. Y. H. Liao, and P.-C. Wang, "Using grey theory in quality function deployment to analyse dynamic customer requirements," The International Journal of Advanced Manufacturing Technology, vol. 25, pp. 1241-1247, 2005.

[7] Y.-E. Nahm, H. Ishikawa, and M. Inoue, "New rating methods to prioritize customer requirements in QFD with incomplete customer preferences," The International Journal of Advanced Manufacturing Technology, vol. 65, pp. 1587-1604, 2012.

[8] X. Chen, C.-H. Chen, K. F. Leong, and X. Jiang, "An ontology learning system for customer needs representation in product development," The International Journal of Advanced Manufacturing Technology, vol. 67, pp. 441-453, 2013.

[9] Z. Sheng, Y. Wang, J. Song, and H. Xie, "Customer requirement modeling and mapping of numerical control machine," Advances in Mechanical Engineering, vol. 7, no. 10, pp. 1-11, 2015.

[10] M. Carulli, M. Bordegoni, and U. Cugini, "An approach for capturing the Voice of the Customer based on Virtual Prototyping,"
Journal of Intelligent Manufacturing, vol. 24, no. 5, pp. 887-903, 2013.

[11] C. C. Aguwa, L. Monplaisir, and O. Turgut, "Voice of the customer: Customer satisfaction ratio based analysis," Expert Systems with Applications, vol. 39, no. 11, pp. 10112-10119, 2012.

[12] E. Ebrahimi, M. Monjezi, M. R. Khalesi, and D. J. Armaghani, "Prediction and optimization of back-break and rock fragmentation using an artificial neural network and a bee colony algorithm," Bulletin of Engineering Geology and the Environment, vol. 75, no. 1, pp. 27-36, 2016.

[13] S. Han, S. Seo, and H. Choi, "A study on modeling customer preferences for conceptual design," Journal of Mechanical Science and Technology, vol. 29, no. 12, pp. 5083-5091, 2015.

[14] M. R. Salehi, L. Noori, and E. Abiri, "Prediction of matching condition for a microstrip subsystem using artificial neural network and adaptive neuro-fuzzy inference system," International Journal of Electronics, vol. 103, no. 11, pp. 1882-1893, 2016.

[15] Y. Juang, S. Lin, and H. Kao, "Design and implementation of a fuzzy inference system for supporting customer requirements," Expert Systems with Applications, vol. 32, no. 3, pp. 868-878, 2007.

[16] X. Wang, B. Sheng, and L. Xue, "Classification of customer requirements on Map Reduce-based Naive Bayes," in Proceedings of the IEEE International Conference on Big Data Analysis, pp. 1-4, 2016.

[17] Y. Wang and M. M. Tseng, "Identifying emerging customer requirements in an early design stage by applying bayes factorbased sequential analysis," IEEE Transactions on Engineering Management, vol. 61, no. 1, pp. 129-137, 2014.

[18] Y. Wang and M. M. Tseng, "A Naïve Bayes approach to map customer requirements to product variants," Journal of Intelligent Manufacturing, vol. 26, no. 3, pp. 501-509, 2015.

[19] P. Zheng and X. Xu, "A weighted interval rough number based method to determine relative importance ratings of customer requirements in QFD product planning," Journal of Intelligent Manufacturing, pp. 1-14, 2016.

[20] M. A. Cun-Bao and M. Zhao, "Application of probability causal network junction tree algorithm in fault diagnosis," Electrical Automation, vol. 23, pp. 12-19, 2014.

[21] P. Kanjohn and A. Grastien, "Local consistency and junction tree for diagnosis of discrete-event systems," Annals of Surgery, vol. 68, pp. 118-126, 2008. 


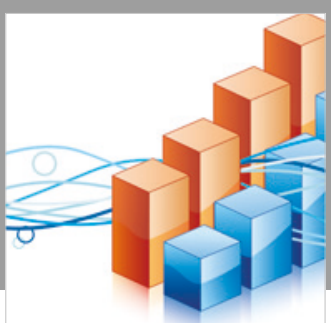

Advances in

Operations Research

\section{-n-m}
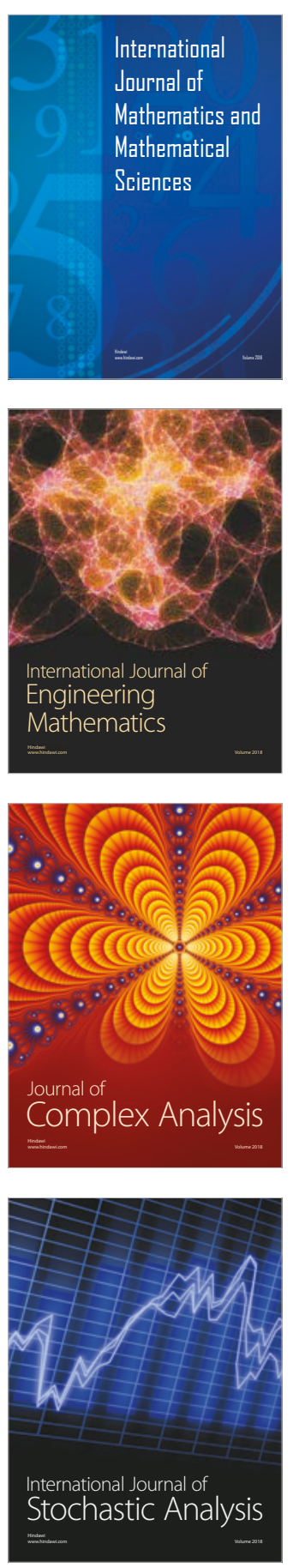
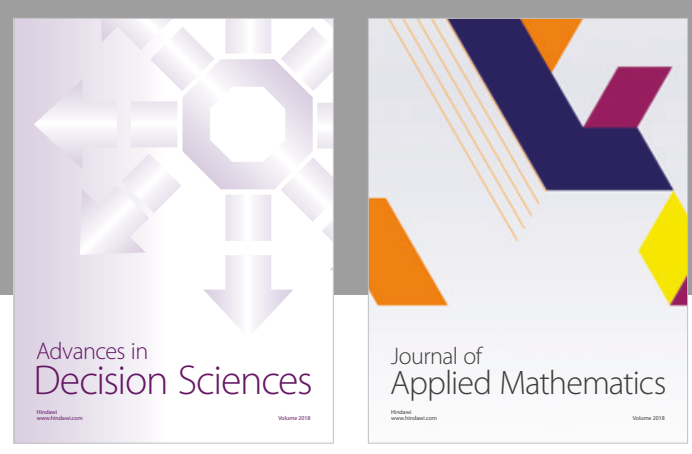

Journal of

Applied Mathematics
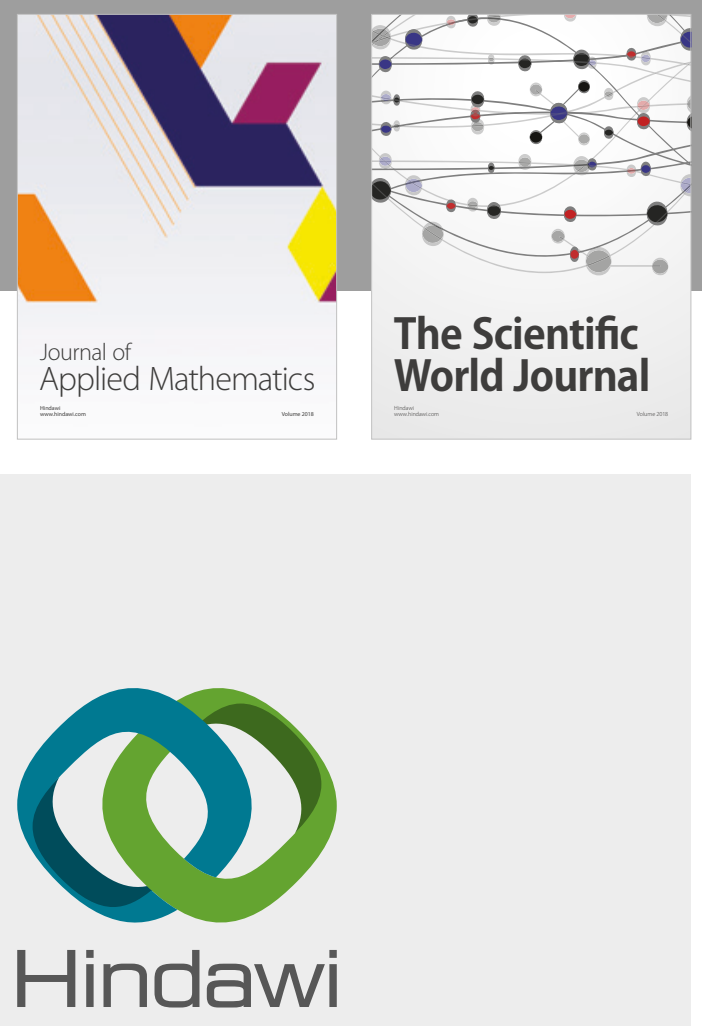

Submit your manuscripts at

www.hindawi.com

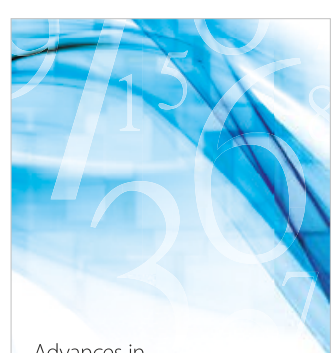

Advances in
Numerical Analysis
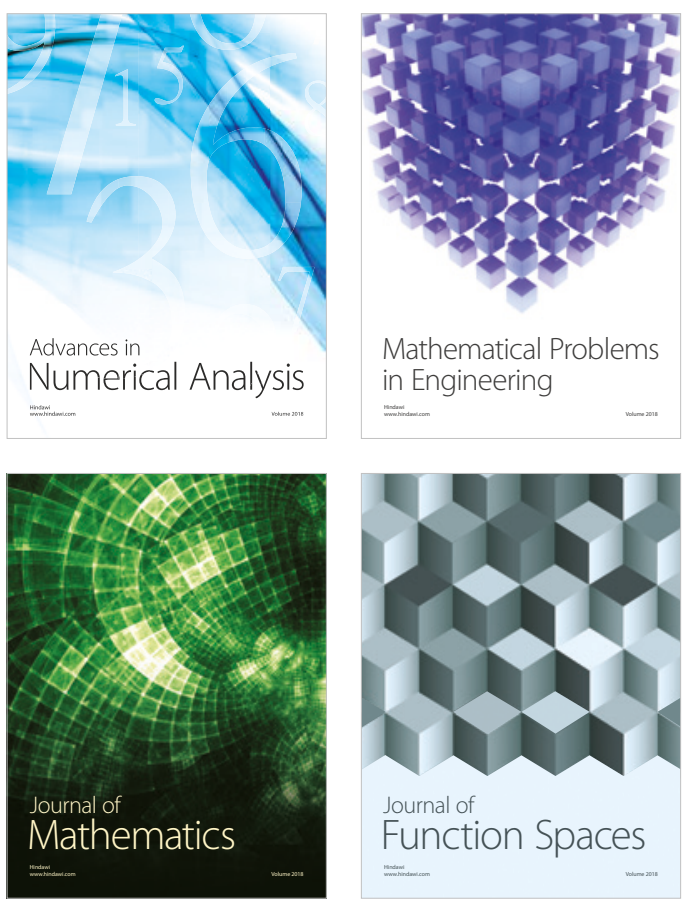

Mathematical Problems in Engineering

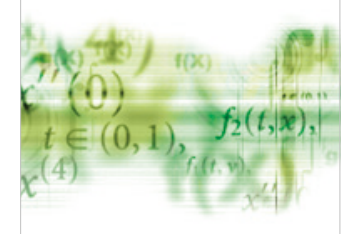

International Journal of

Differential Equations

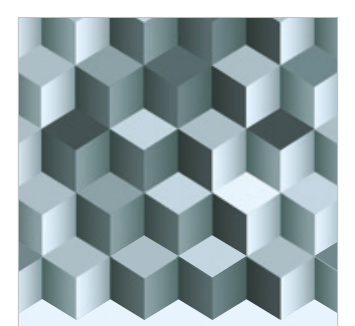

Journal of

Function Spaces

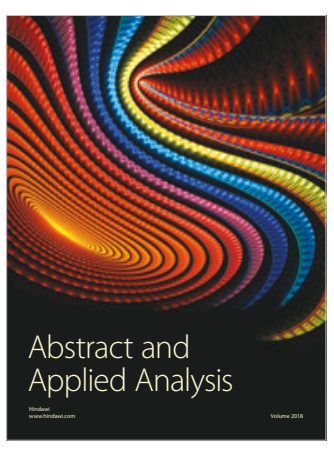

The Scientific

World Journal

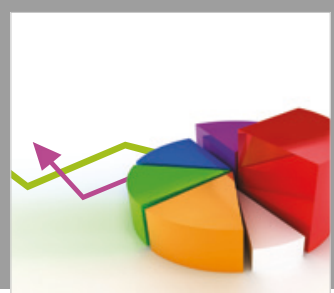

Journal of

Probability and Statistics
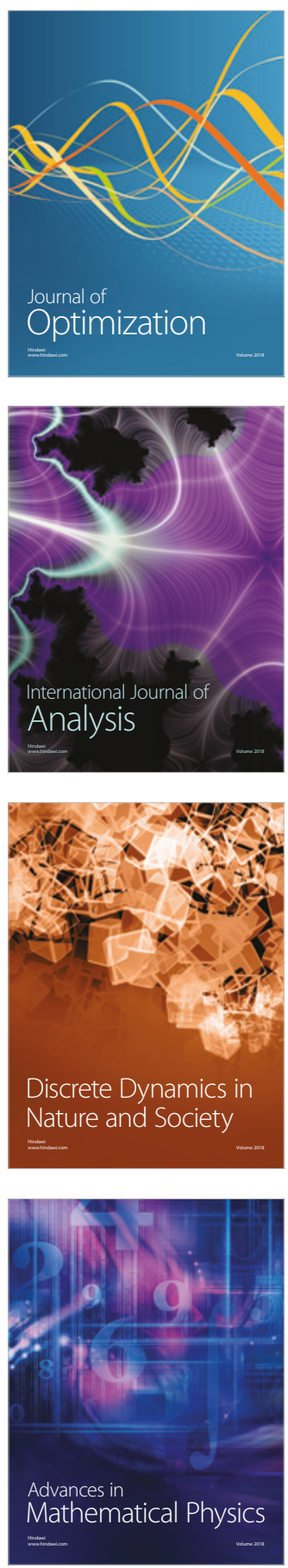